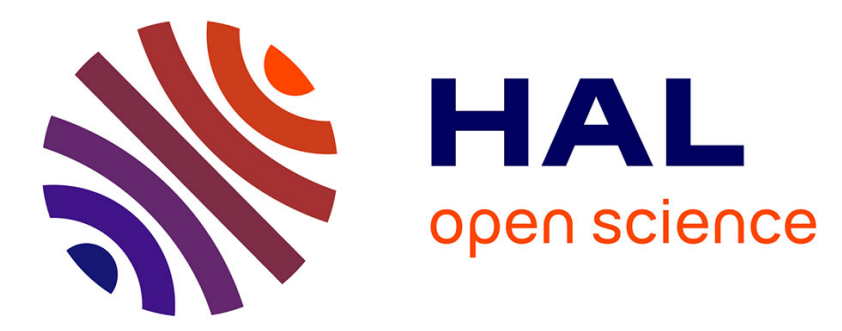

\title{
Modélisation de la barrière de Schottky en présence d'états d'interface distribués spatialement
}

\author{
G.N. Lu, T. Neffati, C. Barret
}

\section{To cite this version:}

G.N. Lu, T. Neffati, C. Barret. Modélisation de la barrière de Schottky en présence d'états d'interface distribués spatialement. Revue de Physique Appliquée, 1987, 22 (12), pp.1809-1817. 10.1051/rphysap:0198700220120180900 . jpa-00245743

HAL Id: jpa-00245743

https://hal.science/jpa-00245743

Submitted on 1 Jan 1987

HAL is a multi-disciplinary open access archive for the deposit and dissemination of scientific research documents, whether they are published or not. The documents may come from teaching and research institutions in France or abroad, or from public or private research centers.
L'archive ouverte pluridisciplinaire HAL, est destinée au dépôt et à la diffusion de documents scientifiques de niveau recherche, publiés ou non, émanant des établissements d'enseignement et de recherche français ou étrangers, des laboratoires publics ou privés. 


\title{
Modélisation de la barrière de Schottky en présence d'états d'interface distribués spatialement
}

\author{
G. N. Lu, T. Neffati et C. Barret \\ Institut d'Electronique Fondamentale, CNRS UA22, Université Paris-Sud, Bât. 220, 91405 Orsay Cedex, \\ France
}

(Reçu le 9 juillet 1987, révisé le 4 septembre 1987, accepté le 7 septembre 1987)

\begin{abstract}
Résumé. - Le profil des bandes d'énergie dans un semiconducteur en contact avec un métal est déterminé en tenant compte d'une charge interfaciale provenant d'états électroniques énergétiquement discrets et de densité décroissant exponentiellement vers le volume du semiconducteur. Le rôle des principaux paramètres (énergie, densité, profondeur de pénétration) d'un état unique, accepteur ou donneur, sur la détermination de la hauteur de barrière est mis en évidence de façon quantitative. Puis, lorsque plusieurs états interviennent simultanément, nous montrons comment construire simplement un diagramme asymptotique décrivant les variations de la hauteur de barrière avec le travail de sortie du métal. Ce modèle est utilisé pour tester les configurations énergétiques plausibles des états intervenant dans les interfaces M-Si, M-GaAs et M-InP.
\end{abstract}

\begin{abstract}
The profile of the energy bands of a semiconductor in contact with a metal is determined by the interface charge distribution due to electronic discrete levels decreasing in density towards the semiconductor bulk. The influence of the states properties (energy position, density and penetration depth) on the Schottky barrier height is quantitatively studied. We show that in the case of more than one level, it is easy to describe the variation in barrier height as a function of the metal work function by means of asymptotic diagrams built from the contribution of each state. The comparison between our model and experimental results on $\mathrm{M}$-Si, $\mathrm{M}$ GaAs and M-InP interfaces is also discussed.
\end{abstract}

\section{Introduction.}

Il apparaît maintenant de plus en plus évident que les interfaces métal-semiconducteur (M-S) réelles ne sont qu'exceptionnellement abruptes au niveau atomique [1]. Il est donc extrêmement probable que la charge interfaciale, généralement reconnue comme jouant un rôle primordial dans la fixation de la barrière de Schottky, ne soit pas strictement localisée dans un hypothétique plan d'interface, mais soit distribuée, côté semiconducteur, sur une distance correspondant à plusieurs couches atomiques.

Quelques auteurs [2-4] ont cherché à intégrer cette donnée dans un modèle électrostatique de la barrière en plaçant cette charge dans un plan situé à quelques angströms sous la surface du semiconducteur. Cette approche très simple a montré que le choix de la position de ce plan avait des conséquences quantitatives importantes en particulier sur les densités d'états nécessaires pour obtenir une hauteur de barrière indépendante du métal. Ceci a été confirmé par une étude plus détaillée de Palau

REVUE DE PHYSIQUE APPLIQUÉE. - T. 22, N` 12, DÉCEMBRE 1987 et al. [5] qui ont utilisé une distribution uniforme d'états dans une couche interfaciale d'épaisseur donnée. Tous ces travaux se sont limités à simuler les variations de la barrière $\phi_{B}$ en fonction du travail de sortie du métal $\phi_{M}$ pour quelques configurations particulières d'états sans effectuer d'étude systématique du rôle des différents paramètres.

Nous nous proposons d'analyser de façon détaillée, à la fois par simulation et par calcul analytique, le comportement d'une interface M-S en présence d'états électroniques énergétiquement discrets et spatialement distribués suivant une loi exponentielle décroissant vers le volume du semiconducteur. Nous chercherons tout d'abord à obtenir le profil des bandes d'énergie dans le semiconducteur. Puis, à partir d'une définition réaliste de la hauteur de barrière et du potentiel de surface du semiconducteur, nous pourrons déterminer la relation $\phi_{B}\left(\phi_{M}\right)$ et préciser quantitativement les conditions de ce qu'on appelle usuellement, et de façon impropre, l'ancrage du niveau de Fermi à l'interface M-S. Ce travail qui a été effectué préalablement dans le cas 
simple d'un seul état d'interface [6] est étendu ici au cas où plusieurs niveaux discrets coexistent. Nous montrerons comment une construction de la courbe $\phi_{B}\left(\phi_{M}\right)$ peut s'effectuer aisément sous forme d'association de diagrammes asymptotiques tant que la profondeur de pénétration des états n'excède pas quelques dizaines d'angströms. Au-delà, quelques exemples de simulation feront apparaître la complexité des profils des bandes d'énergie auxquels on aboutit. Enfin nous comparerons les variations de $\phi_{\mathrm{B}}$ avec $\phi_{\mathrm{M}}$ obtenues avec les résultats expérimentaux disponibles sur $\mathrm{Si}, \mathrm{GaAs}$ et InP.

\section{Description du modèle. Cas d'un état unique.}

La forme des bandes d'énergie dans le semiconducteur est obtenue par intégration de l'équation de Poisson en tenant compte d'une part des densités de charges $\rho_{\mathrm{v}}(x)$ dues aux impuretés de dopage et d'autre part de celles associées à ce que nous continuerons à appeler par commodité les états d'interface. En présence d'états accepteurs et donneurs caractérisés chacun par son niveau d'énergie $E_{\mathrm{s}}$, sa concentration totale $K$ et sa profondeur caractéristique de pénétration $L$, cette densité vaut à une distance $x$ de la surface du semiconducteur:

$$
\begin{aligned}
\rho_{\mathrm{s}}(x)= & -\sum_{j} q \frac{K_{\mathrm{a} j}}{L_{\mathrm{a} j}} \exp \left(-\frac{x}{L_{\mathrm{a} j}}\right) \\
& \times\left\{1+\exp \left[\frac{E_{\mathrm{sa} j}(x)-E_{\mathrm{f}}}{k T}\right]\right\}^{-1} \\
& +\sum_{i} q \frac{K_{\mathrm{d} i}}{L_{\mathrm{d} i}} \exp \left(-\frac{x}{L_{\mathrm{d} i}}\right) \\
& \times\left\{1+\exp \left[\frac{E_{\mathrm{f}}-E_{\mathrm{sd} i}(x)}{k T}\right]\right\}^{-1} .
\end{aligned}
$$

La figure 1 montre les résultats obtenus dans le cas d'un seul état, soit accepteur (Fig. 1a), soit donneur (Fig. 1b), de densité totale $4 \times 10^{13} \mathrm{~cm}^{-2}$, de profondeur de pénétration $10 \AA$ et situé à $0,9 \mathrm{eV}$ de la bande de conduction de GaAs de type $\mathrm{N}$, dopé à $10^{17} \mathrm{~cm}^{-3}$. Ces résultats sont discutés en détail par ailleurs $[6,7]$. Nous rappelons ici les points essentiels.

Il est tout d'abord indispensable de distinguer clairement entre le potentiel à la surface du semiconducteur $\phi(0)$ et la hauteur de barrière $\phi_{\mathrm{B}}$. Le potentiel de surface est lié au travail de sortie du métal suivant la relation de Schottky:

$$
\phi(0)=\phi_{\mathrm{M}}-\chi
$$

où $\chi$ est l'affinité électronique du semiconducteur. En toute rigueur l'équation (2) doit être corrigée d'un terme dipolaire [2] tenant compte de la différence de distribution des électrons de valence des deux matériaux suivant qu'ils sont séparément sous vide ou en contact mutuel. Mais les calculs récents

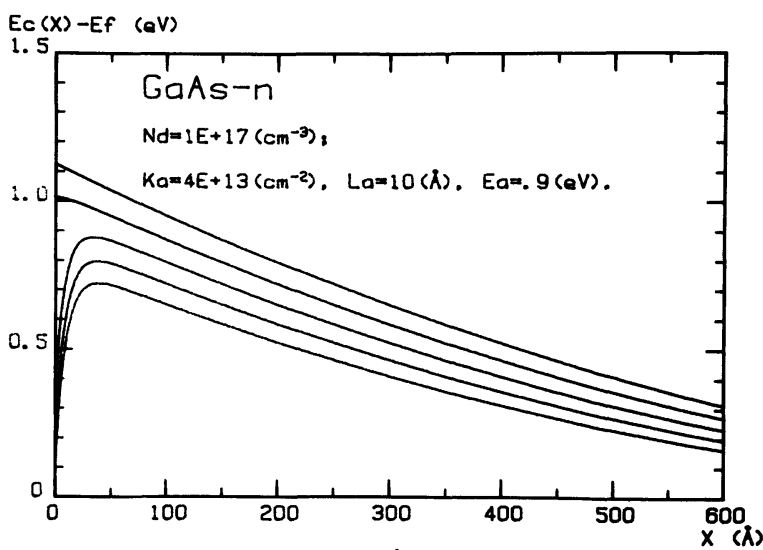

a)

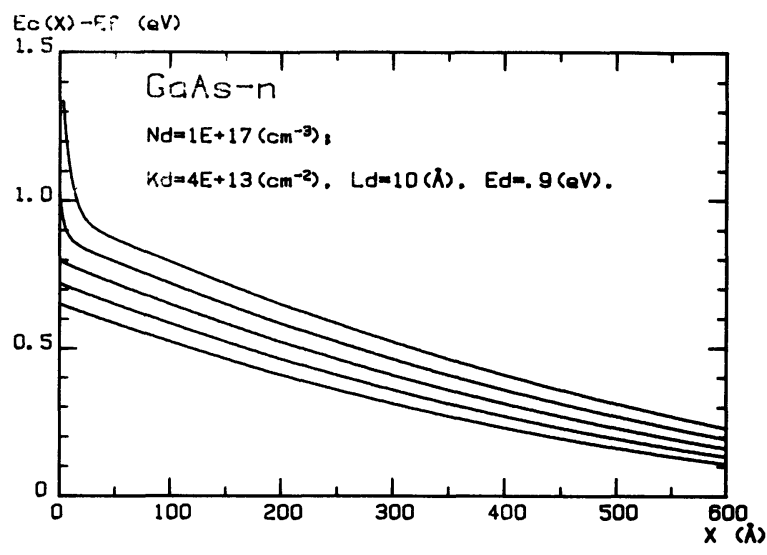

b)

Fig. 1. - Influence d'un état d'interface accepteur (a) ou donneur (b) sur le profil des bandes d'énergie dans GaAs de type n. Les différentes courbes correspondent à différents potentiels de surface, donc à différents métaux.

[Influence of the interface states (in case of an acceptor (a) or a donor (b) level) on the shape of the energy bands for GaAs-n. The various curves correspond to various surface potential, or various metals.]

de Duke et Mailhiot [4] ont montré que ce terme était généralement inférieur à $0,1 \mathrm{eV}$ et nous le négligerons par la suite.

La hauteưr de barrière est clairement définie dans le cas d'un état accepteur par :

$$
\phi_{\mathrm{B}}=\operatorname{Max}\left[E_{\mathrm{c}}(x)-E_{\mathrm{f}}\right] .
$$

En effet la figure 1a montre que le sommet de barrière est enterré dans le semiconducteur à une distance $d$ de la surface. La distance $d$ est définie par :

$$
d=L_{\mathrm{a}} \ln \left(K_{\mathrm{a}} / N_{\mathrm{d}} W\right) .
$$

Cela signifie simplement que la charge négative de l'état pour $x>d$ compense la charge positive $Q_{\text {sc }}$ de la zone de charge d'espace d'épaisseur $W$ due aux atomes dopants ionisés. La couche comprise entre $x=0$ et $x=d$ joue le rôle de zone tampon vis- 
à-vis des variations du travail de sortie du métal. En effet, cette couche subit la différence de potentiel $\phi(0)-\phi_{B}$ qui peut valoir au maximum, lorsque l'accepteur est totalement ionisé et porte une charge très supérieure à $Q_{\mathrm{sc}}$ :

$$
\operatorname{Max}\left[\phi(0)-\phi_{\mathrm{B}}\right]=\Delta \phi_{\mathrm{M}}=\frac{q^{2} K_{\mathrm{a}} L_{\mathrm{a}}}{\varepsilon} .
$$

Cette valeur représente donc la largeur du « palier d'ancrage ", c'est-à-dire du domaine de travail de sortie à l'intérieur duquel la hauteur de barrière ne varie pratiquement pas. Ceci est illustré par la figure 2 qui montre les variations de $\phi_{\mathrm{B}}$ avec $\phi_{\mathrm{M}}$ pour différentes valeurs de $K_{\mathrm{a}}$ et $L_{\mathrm{a}}$.

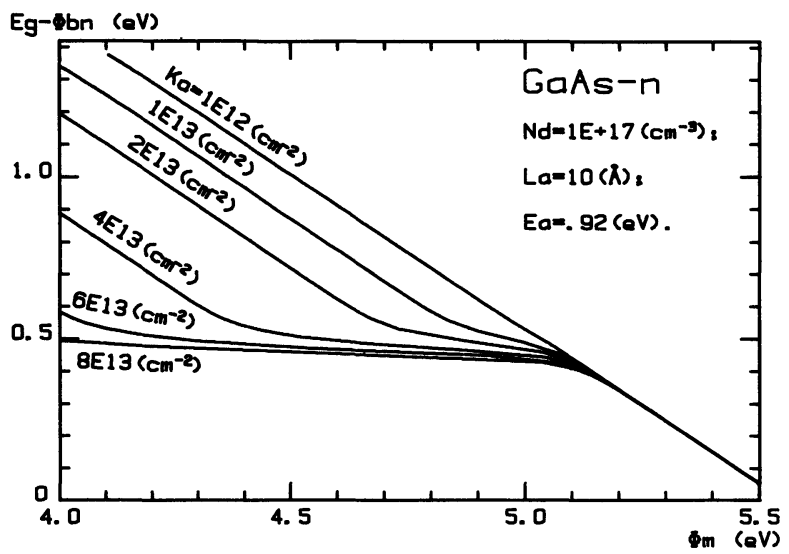

a)

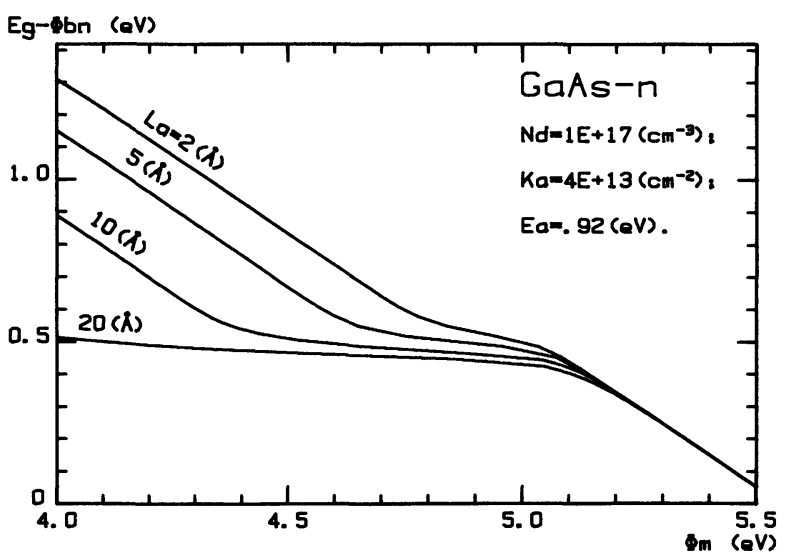

b)

Fig. 2. - Comportement de la hauteur de barrière en fonction du travail de sortie du métal et des paramètres d'un état accepteur: (a) densité ; (b) profondeur de pénétration.

[Behaviour of the barrier height as a function of the metal work function and of parameters of an acceptor state : (a) density ; (b) penetration depth.]

Ces résultats appellent trois remarques. Tout d'abord la relation (5) et la figure 2 démontrent quantitativement que la capacité d'ancrage d'un état dépend à la fois de sa densité et de sa profondeur de pénétration. Ceci est général, quelle que soit la loi de variation de $\rho_{\mathrm{s}}(\chi)$ et confirme les précédents résultats de Palau et coll. [5]. Deuxièmement la nécessité d'une couche isolante interfaciale qui existait dans les modèles à interface abrupte [8] disparaît. Le rôle de cette couche est tout naturellement tenu par la zone tampon. Enfin la courbe $\phi_{B}\left(\phi_{M}\right)$ peut être approximée par un diagramme asymptotique comportant trois parties linéaires (Fig. 3a) :

$$
\left.\begin{array}{ll} 
& \phi_{\mathrm{B}} \approx \phi(0)=\phi_{\mathrm{M}}-\chi \\
\text { pour } \quad \phi_{\mathrm{M}}>\phi_{\mathrm{M} \text { crit }}=\chi+E_{\mathrm{a}} \\
\hline \text { pour } \quad \phi_{\mathrm{B} \text { crit }} \approx E_{\mathrm{a}} \\
\\
-\phi_{\mathrm{B}} \approx \phi_{\mathrm{M}}-\chi-\Delta \phi_{\mathrm{M} \text { crit }}-\Delta \phi_{\mathrm{M}} \\
\text { pour } \quad \phi_{\mathrm{M}}<\phi_{\mathrm{M} \text { crit }}-\Delta \phi_{\mathrm{M}}
\end{array}\right\} .
$$

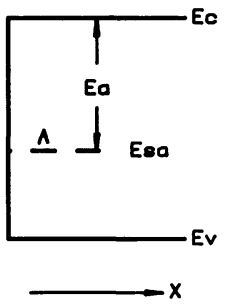

(1)

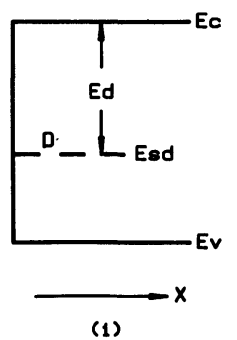

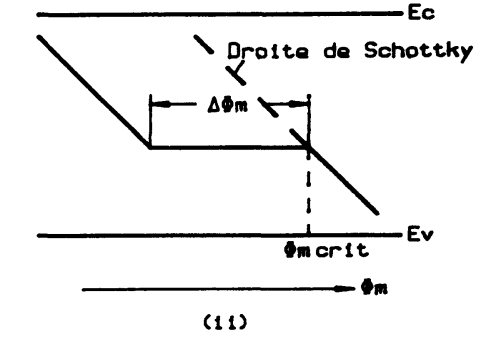

(a)

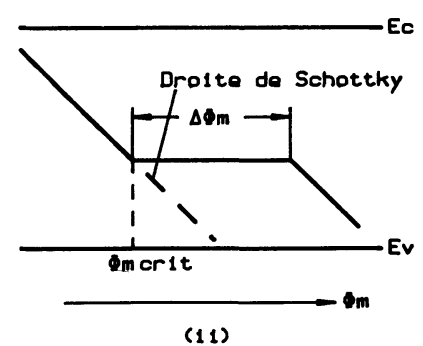

(b)
Fig. 3. - Diagrammes asymptotiques résumant le comportement de $\phi_{\mathrm{B}}\left(\phi_{\mathrm{M}}\right)$ pour un état d'interface unique : (a) accepteur; (b) donneur.

[Asymptotic diagrams describing the behaviour of $\phi_{\mathrm{B}}\left(\phi_{\mathrm{M}}\right)$ in the case of one interface state : (a) acceptor ; (b) donor.]

La première partie correspond à un accepteur neutre car situé au-dessus du niveau de Fermi. La seconde correspond au palier d'ancrage et on doit remarquer qu'il ne s'agit pas en fait d'un blocage du niveau de Fermi à la surface du semiconducteur, mais d'une fixation de $E_{\mathrm{c}}-E_{\mathrm{f}}$ à l'abscisse $d$. Enfin la troisième se produit lorsque l'état, totalement chargé, ne peut plus compenser électrostatiquement la variation de $\phi_{\mathrm{M}}$. 
Considérons maintenant le cas d'un état donneur. La figure $1 \mathrm{~b}$ montre que la barrière présente alors une pointe au voisinage de l'interface. Cette pointe, due à la charge positive de l'état, est à l'évidence traversable par l'effet tunnel. La hauteur de barrière effective n'est donc pas, là encore, à prendre dans le plan d'interface $x=0$. On peut considérer en première approximation que tout se passe comme si une partie d'épaisseur $d_{0}$ de la barrière superficielle était transparente aux électrons, ce qui revient à dire que la barrière effective est :

$$
\phi_{\mathrm{B}}=E_{\mathrm{c}}\left(d_{0}\right)-E_{\mathrm{f}} .
$$

Une détermination précise de $d_{0}$ n'étant ni critique ni indispensable pour la suite, nous nous contenterons d'une estimation grossière de $25 \AA$ pour ce paramètre [7]. Il est par contre intéressant de discuter brièvement des conséquences de la valeur relative de l'épaisseur de la zone tampon par rapport à $d_{0}$. L'épaisseur $d$ de la zone tampon est définie comme pour un accepteur : elle est telle que la charge due à l'état pour $x>d$ soit égale à la charge due au dopage. Si $d$ est inférieur à $d_{0}$, la charge de l'état pour $x>d_{0}$ est négligeable. Dès que le potentiel de surface est assez élevé pour que le niveau de Fermi rencontre le niveau de l'état, celui-ci se charge et provoque la pointe de barrière. La barrière effective à la profondeur $d_{0}$ reste alors pratiquement confondue avec la position de l'état donneur jusqu'à ce que celui-ci, totalement chargé, ait épuisé sa capacité d'ancrage. Les résultats, pour ce qui concerne les variations de $\phi_{\mathrm{B}}$ avec $\phi_{\mathrm{M}}$, sont reportés sur la figure $3 \mathrm{~b}$ sous forme de diagramme asymptotique. La seule différence avec le cas de l'état accepteur est que le palier d'ancrage se trouve maintenant à droite de la droite de Schottky au lieu de se trouver à gauche, c'est-à-dire que ce palier est obtenu pour :

$$
\left.\begin{array}{ll} 
& \phi_{M \text { crit }}<\phi_{M}<\phi_{M \text { crit }}+\Delta \phi_{M} \\
\text { avec } & \phi_{M \text { crit }}=\chi+E_{d} \\
\text { et } & \Delta \phi_{M}=\frac{q^{2} K_{d} L_{d}}{\varepsilon}
\end{array}\right\} .
$$

Si par contre la pénétration de l'état est forte, telle que $d$ devienne supérieur à $d_{0}$, ces résultats ne sont plus valides. Tout d'abord la hauteur de barrière n'est plus égale à la distance séparant l'état de la bande de conduction, mais est donnée par :

$$
\phi_{\mathrm{B}}=E_{\mathrm{d}}+\left|E_{\mathrm{c}}(d)-E_{\mathrm{c}}\left(d_{0}\right)\right| \text {. }
$$

Cela signifie simplement que le niveau de Fermi est à la distance $E_{\mathrm{d}}$ de la bande de conduction à la profondeur $d$, mais que la barrière effective est déterminée à la profondeur $d_{0}$.

Le second point est que la capacité d'ancrage de l'état est réduite d'un facteur $C_{\mathrm{r}}$ tel que :

$$
\begin{aligned}
C_{\mathrm{r}} & =\frac{E_{\mathrm{c}}(0)-E_{\mathrm{c}}\left(d_{0}\right)}{E_{\mathrm{c}}(0)-E_{\mathrm{c}}(d)}=\frac{1-\exp \left(-\frac{d_{0}}{L_{\mathrm{d}}}\right)}{1-\exp \left(-\frac{d}{L_{\mathrm{d}}}\right)} \\
& \approx 1-\exp \left(-\frac{d_{0}}{L_{\mathrm{d}}}\right)
\end{aligned}
$$

Il en résulte que la largeur du palier d'ancrage est donnée par

$$
\Delta \phi_{\mathrm{M}}=C_{\mathrm{r}} \frac{q^{2} K_{\mathrm{d}} L_{\mathrm{d}}}{\varepsilon}
$$

Il est évident que tous les résultats présentés dans ce paragraphe dans le cas d'un matériau de type $n$ sont valides pour un matériau de type $p$ en échangeant le comportement de l'accepteur et du donneur.

\section{Cas de plusieurs états.}

Par association de diagrammes asymptotiques élémentaires relatifs à un seul état, on peut décrire les variations de $\phi_{B}$ avec $\phi_{M}$ lorsque plusieurs états coexistent dans le gap. La figure 4 donne les résultats pour les quatre configurations possibles de deux états accepteurs ou donneurs. Le cas le plus simple est celui où l'état accepteur est situé au-dessus de l'état donneur (Fig. 4a). Il suffit alors d'accoler les deux diagrammes asymptotiques de la figure 3 de part et d'autre de la droite de Schottky. Lorsque deux accepteurs (Fig. 4b) ou deux donneurs sont présents (Fig. 4c) les deux paliers sont soit à gauche, soit à droite de la droite de Schottky. Par exemple dans le cas de deux donneurs, celui qui est situé le plus proche de la bande de conduction va d'abord jouer son rôle d'ancrage lorsque $\phi_{M}-\chi$ atteint la valeur $E_{\mathrm{d} 1}$, comme s'il était seul. Mais le second état n'interviendra que si :

$$
\begin{gathered}
\phi_{\mathrm{M}}>\chi+E_{\mathrm{d} 2}+\Delta \phi_{\mathrm{MD} 1} \\
\Delta \phi_{\mathrm{MD} 1}=\frac{q^{2} K_{\mathrm{d} 1} L_{\mathrm{d} 1}}{\varepsilon}
\end{gathered}
$$

c'est-à-dire que le début du palier qui lui est associé est décalé vers la droite de la quantité $\Delta \phi_{\mathrm{MD} 1}$ due à la présence de l'état $D_{1}$ totalement chargé positivement.

De la même façon, pour deux accepteurs (Fig. 4b), c'est le plus profond, $\left(\mathrm{A}_{2}\right)$, qui conserve son diagramme asymptotique identique à ce qui est obtenu lorsqu'il est seul. L'état $\mathrm{A}_{1}$ situé plus près de la bande de conduction aura son palier décalé vers la gauche de $\Delta \phi_{\text {MA2 }}$ à cause de la charge négative de $A_{2}$. Enfin si un état donneur est au-dessus d'un état accepteur (Fig. 4d) la construction de la courbe asymptotique s'effectue de la manière suivante. Pour les faibles valeurs de $\phi_{M}$ l'accepteur reste totalement chargé. Le diagramme relatif à l'état 

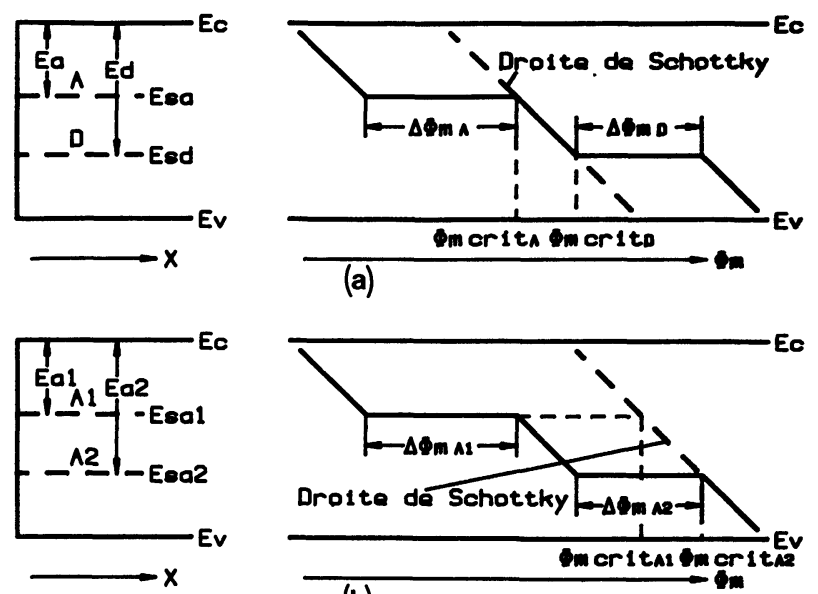

(b)
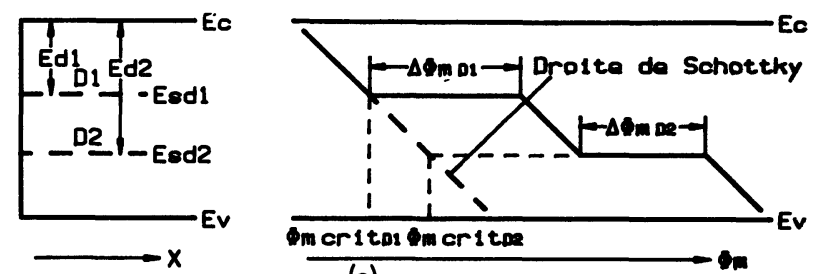

(c)
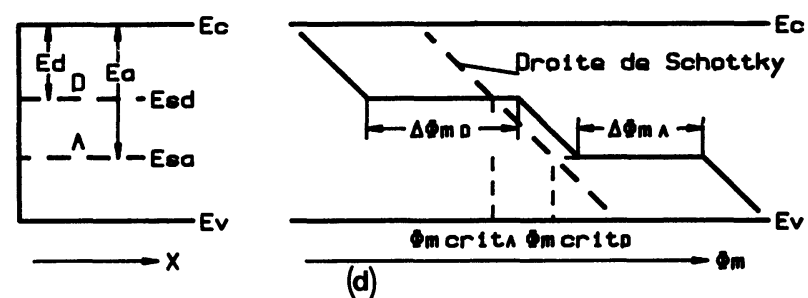

Fig. 4. - Description asymptotique de $\phi_{B}\left(\phi_{M}\right)$ pour les quatre combinaisons possibles de deux états d'interface.

[Asymptotic description of $\phi_{B}\left(\phi_{M}\right)$ for the four possible combinations of two interface states.]

donneur est donc décalé vers la gauche de $\Delta \phi_{\mathrm{MA}}$. Puis lorsque le niveau de Fermi se trouve entre les deux états, ils sont tous les deux totalement chargés. Le diagramme relatif à l'état accepteur sera décalé vers la droite ou vers la gauche de la quantité $\Delta \phi_{\mathrm{MD}}-\Delta \phi_{\mathrm{MA}}$ suivant le signe de la charge résultante.

On peut étendre ces règles d'association à un nombre d'états plus élevé en tenant compte, pour chacun, du décalage à introduire dans le placement du palier qui lui est associé par la charge globale due aux autres états.

La validité de cette approche simple est cependant limitée aux cas où les profondeurs de pénétration des états sont faibles, inférieures à quelques dizaines d'angströms, comme nous l'avons signalé au paragraphe 2. Si un ou plusieurs états s'enfoncent profondément dans le semiconducteur, une simulation complète est indispensable car le profil de la barrière peut être très complexe. Ceci est illustré par quelques exemples sur la figure 5. Le premier cas (Fig. 5a) est celui d'un état donneur de forte densité

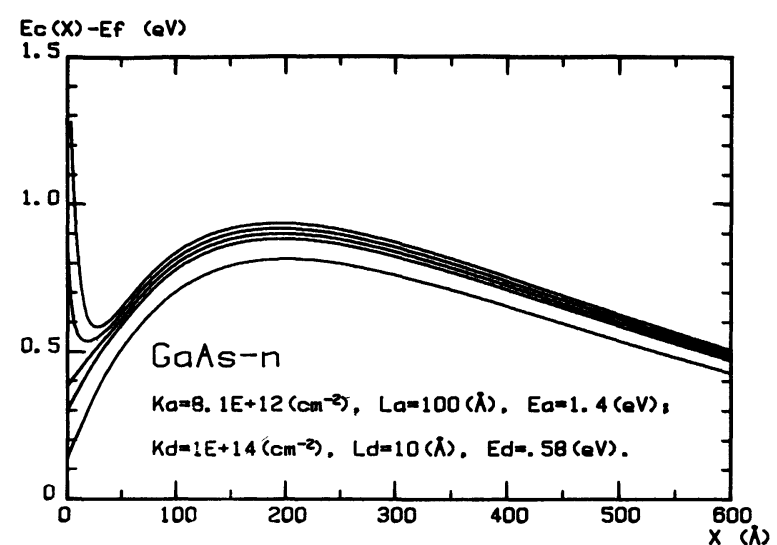

a)

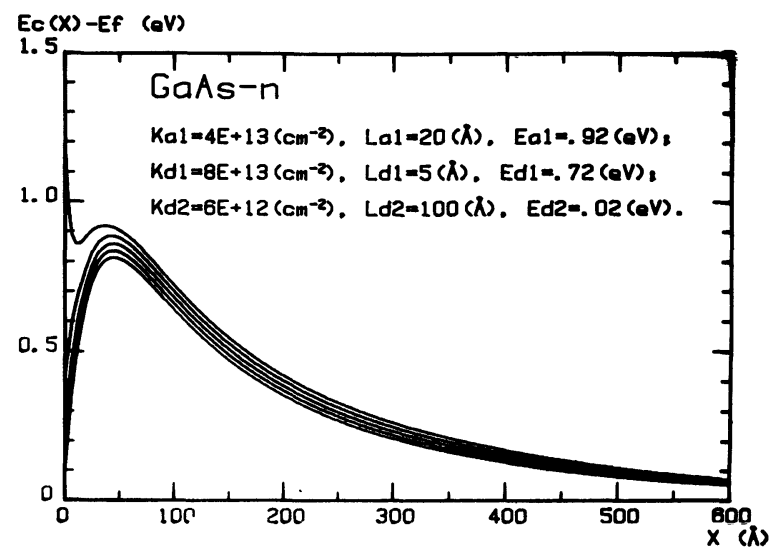

b)

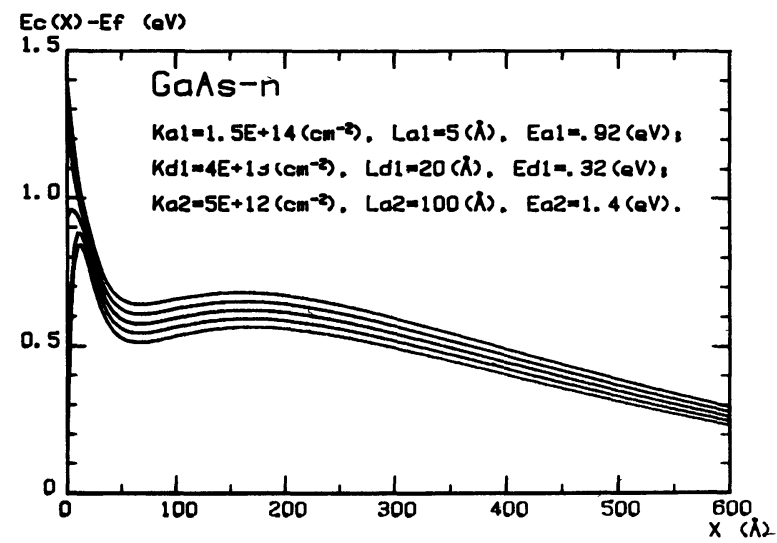

c)

Fig. 5. - Exemples de déformations de profil de la barrière associées à des fortes profondeurs de pénétration des états d'interface.

[Examples for the case of deep penetrations of the interface states which give rise to various distortions of the barrier shape.]

et de faible profondeur de pénétration énergétiquement situé vers le milieu du gap, associé à un accepteur de faible densité, de forte pénétration et situé au voisinage de la bande de valence. On constate la forme complexe de la barrière de potentiel, en particulier pour les forts travaux de sortie 
métalliques. Cependant la hauteur de barrière effective, qui est imposée par l'extremum profondément enterré dans le semiconducteur est quasiment indépendante du métal. Si on regarde la valeur du palier d'ancrage on constate qu'il ne correspond à aucun des deux niveaux énergétiques des états. Ceci s'explique de la façon suivante : l'état donneur provoque une fixation du potentiel à son niveau, à quelques angströms sous la surface. Mais l'état accepteur, totalement chargé, décale ensuite la barrière qui est donnée par :

$$
\phi_{\mathrm{B}}=E_{\mathrm{d}}+\frac{q^{2} K_{\mathrm{a}} L_{\mathrm{a}}}{\varepsilon}
$$

Dans le deuxième exemple (Fig. 5b) trois états sont présents : un donneur $\mathrm{D}_{1}$ à faible concentration, forte pénétration, situé près de la bande de conduction, un donneur $\mathrm{D}_{2}$ et un accepteur $\mathrm{A}_{1}$ situés vers le milieu du gap et de densité et profondeur de pénétrations moyennes. On obtient dans ce cas un sommet de barrière dont l'abscisse est imposée par l'accepteur mais dont l'amplitude évolue entre $E_{\mathrm{a} 1}$ et $E_{\mathrm{d} 2}$.

Enfin, dans le troisième cas (Fig. 5c), un accepteur $\mathrm{A}_{1}$ à forte pénétration impose, comme dans le premier exemple, un premier extremum très loin de l'interface, puis les autres états $A_{2}$ et $D_{1}$ provoquent un second extremum très près de la surface. La barrière effective peut «sauter » d'un extremum à l'autre suivant que la barrière superficielle peut ou non être considérée comme transparente aux électrons. Il est alors illusoire de chercher une quelconque règle simple pour estimer le comportement de $\phi_{\mathrm{B}}$ en fonction de $\phi_{\mathrm{M}}$. On peut aussi remarquer que de telles formes de barrière doivent conduire à des différences de hauteur très importantes suivant la technique de mesure employée $(I-V$ ou $C-V)$ et que de fortes dispersions des résultats sont à attendre si de légères fluctuations des paramètres associés aux états se produisent.

De tels comportements ne sont pas de simples curiosités car des indices expérimentaux montrent que des interfaces réelles peuvent les suivre. Il est connu par exemple que les hauteurs de barrière mesurées sur des diodes Al-InP varient considérablement d'un auteur à l'autre [9-11]. Des profils de bandes du type de ceux de la figure 5 peuvent expliquer ces dispersions, d'autant plus que la présence de défauts s'étendant très loin de l'interface a été mise en évidence dans les contacts Al-InP [12]. Un autre type d'interfaces réelles susceptibles de posséder des états à pénétration profonde peut être obtenu en nettoyant la surface du semiconducteur par bombardement ionique de basse énergie. De très nombreuses études ont été faites $[13,14]$ sur des contacts de ce type et montrent des comportements anormaux des caractéristiques électriques comme on peut s'y attendre à partir des résultats de la figure 5 .

\section{Comparaison avec des résultats expérimentaux.}

Malgré quelques progrès récents [15] les informations expérimentales quantitatives précises sur les états d'interface sont encore très insuffisantes pour pouvoir en déduire directement des règles d'évolution des hauteurs de barrière. La seule démarche possible est alors de partir de courbes expérimentales $\phi_{\mathrm{B}}\left(\phi_{\mathrm{M}}\right)$ et de rechercher à partir de notre modèle les distributions d'états compatibles. Trois remarques préalables sont toutefois à prendre en compte. Tout d'abord il existe une dispersion non négligeable des valeurs publiées de $\phi_{\mathrm{M}}$ et de $\phi_{\mathrm{B}}$. Ceci n'est pas très critique pour $\phi_{M}$ en raison de l'échelle relativement étendue des valeurs, et nous avons retenu une moyenne entre plusieurs résultats [16-18]. Le problème est plus délicat pour $\phi_{\mathrm{B}}$ car ces dispersions peuvent masquer l'influence du métal lorsque celleci est faible [19]. Nous avons écarté toute valeur publiée sans que les conditions expérimentales soient clairement indiquées et nous n'avons conservé que les résultats provenant, soit de mesures photoélectrique, soit de mesures $I-V$ afin que les barrières s'approchent le plus possible de la définition que nous avons choisie. Le second point est que beaucoup de métaux étudiés de façon intensive correspondent à des travaux de sortie compris typiquement entre 4 et $5 \mathrm{eV}$, alors qu'on dispose de peu de résultats pour des valeurs de $\phi_{\mathrm{M}}$ plus élevées et surtout plus faibles. Ceci peut rendre difficile la mise en évidence de points de cassure dans les courbes $\phi_{\mathrm{B}}\left(\phi_{\mathrm{M}}\right)$. Enfin les simulations effectuées montrent qu'une courbe $\phi_{B}\left(\phi_{M}\right)$ donnée peut être obtenue à partir de plusieurs distributions différentes d'états. Nous adopterons alors la méthodologie suivante : partir des cas les plus simples (un seul état, deux états, ...) et déterminer s'ils sont plausibles ou non en cernant au mieux les points critiques des comportements théoriques et expérimentaux, c'est-à-dire, en particulier, la position des points de cassure des courbes $\phi_{\mathrm{B}}\left(\phi_{\mathrm{M}}\right)$.

La figure 6 donne les points expérimentaux $\phi_{\mathrm{B}}\left(\phi_{\mathrm{M}}\right)$ pour Si, GaAs et InP clivés sous ultra-vide. Nous discuterons tout d'abord du cas du silicium pour lequel nous disposons du plus grand nombre de résultats expérimentaux. Il apparaît clairement que les points ne sont pas distribués suivant une simple fonction linéaire [8], mais suivent le comportement asymptotique indiqué en pointillé sur la figure $6 \mathrm{a}$ : deux paliers à $\phi_{\mathrm{B}}=0,4 \mathrm{eV}$ et $0,8 \mathrm{eV}$, reliés par une partie à croissance rapide, de pente unité entre $\phi_{M}=3,6$ et 4,0 eV. Si nous comparons ces résultats avec les cas modélisés précédemment, il est évident que deux états (au moins) participent au blocage du niveau de Fermi. Si nous éliminons par simplicité le cas de pénétrations profondes qui induiraient des comportements anormaux, nous en concluons qu'un accepteur ou'un donneur se trouvent au voisinage de 


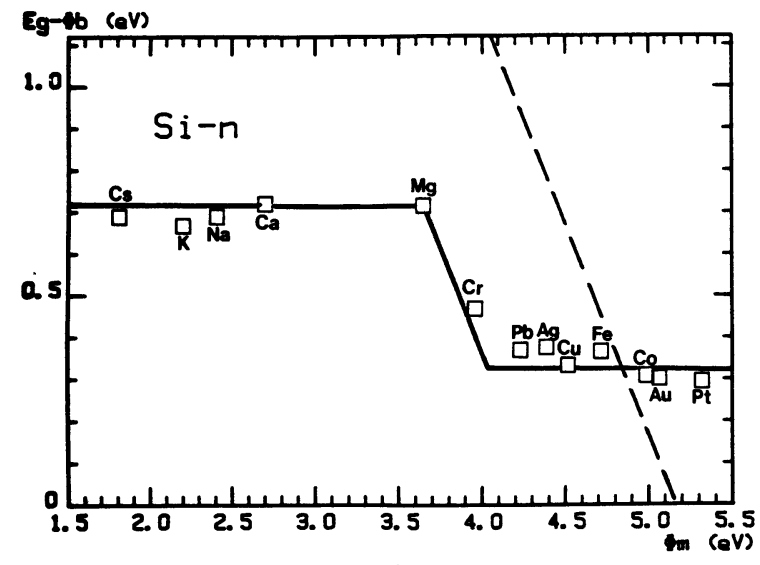

a)

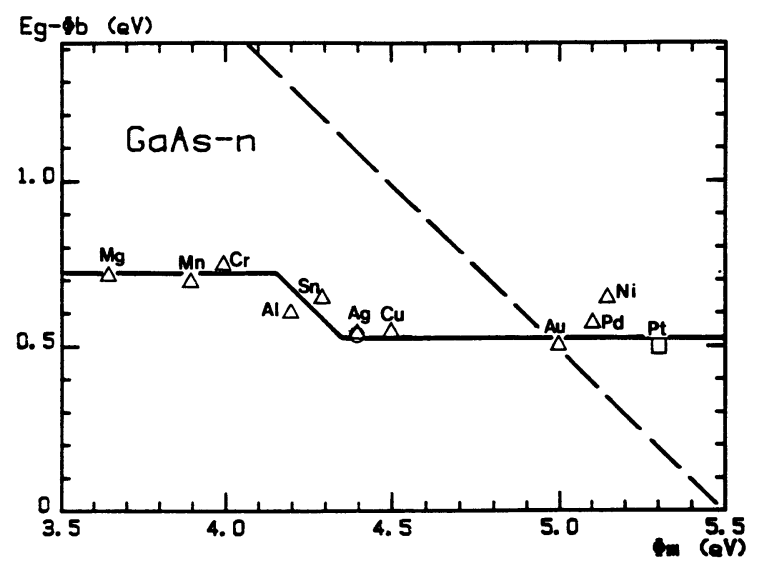

b)

$E_{\mathrm{c}}-0,4 \mathrm{eV}$ et de $E_{\mathrm{c}}-0,8 \mathrm{eV}$. Pour préciser la nature de ces états il faut analyser les valeurs numériques des positions des points de cassure. Si on se reporte à la figure 4 on constate tout d'abord que la position du diagramme par rapport à la droite de Schottky interdit les situations correspondant à deux donneurs ou à un accepteur au-dessus d'un donneur. Dans l'un ou l'autre de ces deux cas un troisième état, au moins, serait indispensable. Cet état doit être chargé négativement, donc de type accepteur et placé plus profondément dans le gap que les deux autres.

On peut même préciser dans le cas d'une distribution accepteur-donneur-accepteur, que ce second accepteur $A_{2}$ doit être tel que :

$$
\Delta \phi_{\mathrm{MA} 2}=\frac{q^{2} K_{\mathrm{a} 2} L_{\mathrm{a} 2}}{\varepsilon} \approx 0,85 \mathrm{eV} .
$$

Le cas de deux accepteurs seuls n'est pas non plus possible car une croissance de $\phi_{\mathrm{B}}$ devrait se manifester au-delà de la valeur de $\phi_{\mathrm{M} \text { crit }}$ de l'état le plus profond, soit $4,85 \mathrm{eV}$ d'après la relation (6). L'absence d'une telle croissance pourrait être due à un troisième état, donneur positivement chargé, provoquant un décalage du diagramme vers la droite. Seul le cas d'un donneur $\mathrm{D}_{1}$ situé au-dessus

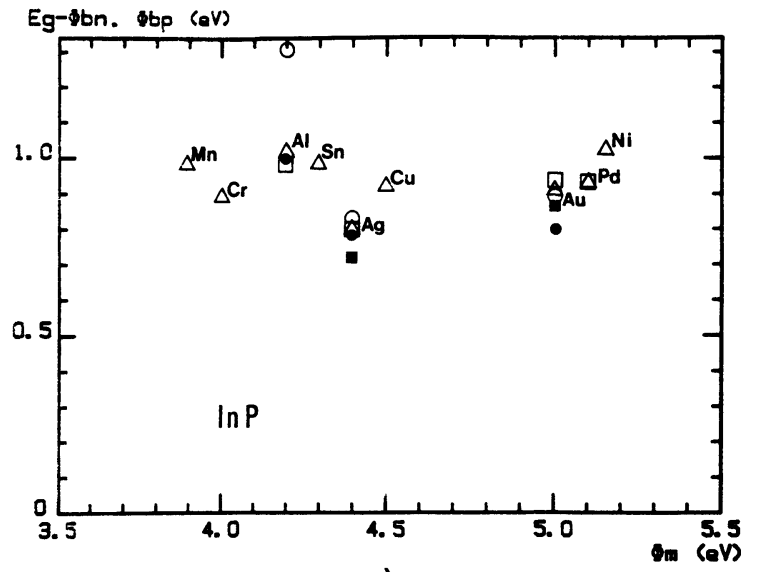

c)

Fig. 6. - Compilation de résultats expérimentaux de la hauteur de barrière sur: (a) silicium (de type $n$ ), $\square$ : Barret et al. [18] ; (b) GaAs, $\Delta$ : Newman et al. [22], $\square$ : Barret et al. [23] ; (c) InP, symboles vides pour type n,

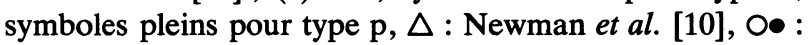
Lassabatère et al. [11], $\square$ : nos mesures. Le copportement asymptotique de $\phi_{B}\left(\phi_{M}\right)$ ( $(-)$ et la droite de Schottky ( --$)$ sont indiqués sur (a) et (b).

[Compilation of experimental results of the barrier heights for: (a) silicon (of $\mathrm{n}$ type), $\square$ : Barret et al. [18] ; (b) GaAs, $\triangle$ :Newman et al. [22], $\square$ : Barret et al. [23] ; (c) InP, empty symbols for $\mathbf{n}$ type and solid symbols for $\mathbf{p}$ type, $\Delta$ : Newman et al. [10], O• : Lassabatère et al. [11], : our measurements. The asymptotic curves $\phi_{\mathrm{B}}\left(\phi_{\mathrm{M}}\right)$ $(-)$ and the Schottky line ( $-\longrightarrow$ ) are given on (a) and (b).]

d'un accepteur $A_{1}$ peut expliquer quantitativement les résultats expérimentaux sans faire appel à un troisième état. La position des points de cassure implique que :

$$
\begin{aligned}
& \chi+E_{\mathrm{a}}+\Delta \phi_{\mathrm{MD}}-\Delta \phi_{\mathrm{MA}}=4,0 \mathrm{eV} \\
& \chi+E_{\mathrm{d}}+\Delta \phi_{\mathrm{MD}}-\Delta \phi_{\mathrm{MA}}=3,6 \mathrm{eV}
\end{aligned}
$$

soit :

$$
\Delta \phi_{\mathrm{MA}}-\Delta \phi_{\mathrm{MD}}=0,8 \mathrm{eV}
$$

ce qui conduit à :

$$
K_{\mathrm{a}} L_{\mathrm{a}}-K_{\mathrm{d}} L_{\mathrm{d}}=5,5 \times 10^{6} \mathrm{~cm}^{-1} .
$$

D'autre part les longueurs des paliers expérimentaux impliquent que :

$$
\begin{aligned}
& K_{\mathrm{d}} L_{\mathrm{d}} \geqslant 1,1 \times 10^{7} \mathrm{~cm}^{-1} \\
& K_{\mathrm{a}} L_{\mathrm{a}} \geqslant 7,5 \times 10^{6} \mathrm{~cm}^{-1} .
\end{aligned}
$$

Cette dernière relation étant automatiquement remplie si les deux précédentes le sont. Notons que les valeurs de $K L$ obtenues correspondent à des densités d'états assez élevées : si on se limite à des valeurs de $L$ de l'ordre de $10 \AA$, il est nécessaire que $K \approx 10^{14} \mathrm{~cm}^{-2}$ pour obtenir des valeurs de $K L$ autour de $10^{7} \mathrm{~cm}^{-1}$. Une comparaison plus fine avec 
des résultats expérimentaux serait sans objet. Notons cependant que les études effectuées sur les états d'interfaces [18] montrent l'existence de bandes étroites d'états dans le gap, l'une située au-dessous de $E_{\mathrm{c}}-0,4 \mathrm{eV}$ et l'autre en dessous de $E_{\mathrm{c}}-0,8 \mathrm{eV}$ en parfait accord avec nos déterminations. La densité de ces états semble varier d'un métal à l'autre, ce qui n'a pas été pris en compte dans nos calculs, mais ne change pas fondamentalement nos conclusions. Il est à remarquer aussi que Muret $[20,21]$ observe, pour des interfaces $\mathrm{Au}-\mathrm{Si}$ et $\mathrm{Ni}$-Si, des densités apparentes d'états élevées qu'il interprète par une répartition dans une zone de plusieurs couches atomiques.

On dispose sur GaAs de moins de mesures de barrière que sur le silicium en particulier pour les métaux à faible travail de sortie. Nous avons retenu sur la figure $6 \mathrm{~b}$ les valeurs les plus probables de $\phi_{\mathrm{B}}\left(\phi_{\mathrm{M}}\right)$ pour une dizaine de métaux. Nous voyons que, mis à part le cas du nickel, les points expérimentaux sont, comme pour $\mathrm{Si}$, répartis sur trois zones : un palier autour de $0,70 \mathrm{eV}$ pour $\phi_{\mathrm{M}}<4,05 \mathrm{eV}$, un palier autour de $0,90 \mathrm{eV}$ pour $\phi_{\mathrm{M}}>4,3 \mathrm{eV}$ et une transition linéaire entre ces deux paliers. Les conclusions sont semblables à celles que nous avons obtenues pour le silicium. Le cas d'un seul état est automatiquement exclu en raison de l'existence de deux paliers. Deux états ne peuvent expliquer le comportement expérimental que si un donneur $D_{1}$ est au-dessus d'un accepteur $A_{1}$ et si :

$$
K_{\mathrm{a}} L_{\mathrm{a}}-K_{\mathrm{d}} L_{\mathrm{d}}=5 \times 10^{6} \mathrm{~cm}^{-1}
$$

et

$$
K_{\mathrm{d}} L_{\mathrm{d}} \geqslant 7 \times 10^{6} \mathrm{~cm}^{-1}
$$

Cette solution, la plus simple, est cohérente avec les résultats obtenus par Spectroscopie de Capacité Schottky sur des diodes $\mathrm{Au}, \mathrm{Ag}$ et Al-GaAs [15, 24, 25] qui montrent l'existence de deux états autour de $E_{\mathrm{c}}-0,7 \mathrm{eV}$ et $E_{\mathrm{c}}-0,9 \mathrm{eV}$. De plus le rapport élevé entre la section efficace de capture des électrons et celles des trous [24] pour l'état situé en $E_{\mathrm{c}}-0,7 \mathrm{eV}$ incite à lui attribuer le type donneur. Cependant une autre solution est également attractive. L'étude des surfaces clivées sous ultra-vide et recouvertes de dépôts métalliques ultra-minces a conduit Spicer et al. [26] à proposer l'existence d'un état accepteur vers $E_{\mathrm{c}}-0,7 \mathrm{eV}$ et d'un donneur en $E_{\mathrm{c}}-0,9 \mathrm{eV}$. Ce résultat ne donne pas un diagramme asymptomique en accord avec les résultats expérimentaux pour des métallisations épaisses. Il faut qu'un second accepteur profond chargé et tel que $K_{\mathrm{a} 2} L_{\mathrm{a} 2} \approx$ $5 \times 10^{6} \mathrm{~cm}^{-1}$ soit présent pour translater la courbe théorique vers la gauche des $0,7 \mathrm{eV}$ nécessaire. Or Skeath et al. [27] ont effectivement suggéré qu'à partir d'un certain taux de couverture un nouveau mécanisme fait apparaître un niveau accepteur supplémentaire proche de la bande de valence. Ce résultat serait alors en accord avec nos simulations.
Pour les interfaces M-InP on ne dispose que d'un faible nombre de mesures de hauteur de barrière fiables. La figure $6 c$ regroupe quelques mesures récentes. Le résultat est un nuage de points au milieu desquels il est bien difficile de déceler un comportement global. Cela ne signifie pas forcément que le modèle que nous avons développé ne s'applique pas. En effet, le matériau InP est beaucoup plus fragile que Si et GaAs. Des études d'intensité de photoluminescence montrent que la surface d'InP est très sensible à la nature d'ions métalliques adsorbés. Il semble que les ions tels que $\mathrm{Ag}^{+}$, $\mathrm{Cu}^{+}, \mathrm{Fe}^{+}$provoquent la création d'un état accepteur vers le milieu du gap, tandis que d'autres ions métalliques n'ont pas le même effet. Ceci montre que, pour InP, les distributions énergétiques et géométriques des états aussi bien que leurs densités peuvent être fortement affectées par la nature des atomes déposés ou adsorbés. Dans ce cas, l'échelle horizontale n'a plus de signification précise puisque les caractéristiques des courbes asymptotiques que nous avons établies varient d'un métal à l'autre. De plus, si nous avons négligé pour $\mathrm{Si}$ et $\mathrm{GaAs}$ la possibilité de grandes profondeurs de pénétration pour la charge interfaciale, cela n'est pas forcément acceptable pour InP. On peut, par exemple, citer Slowik et al. [12] qui expliquent le comportement anormal des courbes $C-V$ sur Al-InP clivé sous ultravide par un état accepteur proche de la bande de conduction et pénétrant jusqu'à $200 \AA ̊$. Ceci veut dire qu'on ne peut même pas assurer que ce sont des états d'énergie comprise entre $E_{\mathrm{c}}-0,35 \mathrm{eV}$ et $E_{\mathrm{c}}-0,55 \mathrm{eV}$ qui fixent la hauteur de barrière.

\section{Conclusion.}

Nous avons étudié de façon quantitative détaillée le rôle joué par les états d'interface dans la fixation de la barrière de Schottky. Le modèle utilisé a été établi avec la volonté de serrer le plus près possible la réalité physique. Les hypothèses de base sont que les états d'interfaces sont discrets et possèdent une charge qui décroît exponentiellement vers le volume du semiconducteur et que la barrière de Schottky doit être évaluée de façon réaliste en tenant compte de la possibilité d'effet tunnel à travers des barrières très fines.

L'accent a été mis sur la dépendance de la hauteur de barrière $\phi_{\mathrm{B}}$ au travail de sortie du métal $\phi_{M}$. Nous montrons qu'un seul état, de type accepteur ou donneur, suffit pour obtenir un ancrage du niveau de Fermi sur une plage de travaux de sortie qui dépend du produit de la concentration totale de l'état par sa profondeur caractéristique de pénétration dans le semiconducteur. L'ancrage cependant peut ne pas se produire autour du niveau énergétique de l'état dans le cas d'un donneur (accepteur) pénétrant relativement profondément dans un matériau de type $N(P)$. 
Lorsque plusieurs états coexistent dans le gap, nous avons montré qu'il était possible de construire aisément les courbes $\phi_{B}\left(\phi_{M}\right)$ dues à l'existence de plusieurs états distincts à partir des diagrammes asymptotiques caractéristiques d'un état accepteur ou d'un état donneur. Cependant, nos simulations montrent que la validité de cette démarche diminue lorsque la profondeur de pénétration d'un ou des états augmente. On peut également obtenir, dans ce cas, des paliers d'ancrage de la hauteur de barrière distincts des positions réelles des états.

Une comparaison quantitative de ce modèle avec les résultats expérimentaux sur les principales interfaces M-S a été effectuée. Cependant, les difficultés sont grandes à cause de l'imprécision des informations expérimentales et de la non-unicité des solu- tions. C'est pourquoi nous nous sommes limités à tester la possibilité d'expliquer les courbes $\phi_{B}\left(\phi_{M}\right)$ expérimentales par les distributions d'états les plus simples possibles.

Dans le cas de Si et GaAs deux états ne suffisent à expliquer les résultats expérimentaux qu'à condition qu'ils soient de type différents, le donneur étant situé au-dessus de l'accepteur. Deux états de même type ou un accepteur placé au-dessus d'un donneur ne peuvent donner un accord quantitatif avec les points expérimentaux. Un troisième état est indispensable pour y parvenir. Enfin, dans le cas d'InP, la dispersion des résultats peut provenir d'une sensibilité beaucoup plus grande des paramètres caractéristiques des états à la nature du métal et aux conditions expérimentales de son dépôt.

\section{Bibliographie}

[1] Brillson, L. J., Surf. Sci. Rep. 2 (1982) 1.

[2] Zur, A., McGill, T. C., Smith, D. L., Phys. Rev. B 28 (1983) 2060.

[3] Spicer, W. E., Pan, S., Mo, D., Newman, N., Mahowald, P., Kendelewicz, T., Eglash, S., J. Vac. Sci. Technol. B 2 (1984) 476.

[4] Duke, C. B., Mailhiot, C., J. Vac. Sci. Technol. B 3 (1985) 1170.

[5] Palau, J. M., Ismail, A., Lassabatère, L., SolidState Electron. 28 (1985) 499.

[6] Lu, G. N., Barret, C., Neffati, T., Revue Phys. Appl., à paraître.

[7] Lu, G. N., Thèse de Doctorat, Orsay (1986).

[8] Cowley, A. M., Sze, S. M., J. Appl. Phys. 36 (1965) 3212.

[9] Williams, R. H., Varma, R. R., MacKinley, A., J. Phys. C 10 (1977) 4545.

[10] Newman, N., Kendelewicz, T.,Bowman, L., SpICER, W. E., Appl. Phys. Lett. 46 (1985) 1176.

[11] Lassabatère, L., Ismail, A., Palau, J. M., BraHIM, A. B., Surf. Sci. 168 (1986) 356.

[12] SlowiK, J. H., Richter, H. W., Brillson, L. T., J. Appl. Phys. 58 (1985) 3154.

[13] Mullins, F. H., Brunnschweiler, A., Solid-State Electron. 19 (1976) 47.
[14] Kwan, P., Bhat, K. N., Borrego, J. M., Gandhi, S. K., Solid-State Electron. 26 (1983) 125.

[15] Chekir, F., Barret, C., Surf. Sci. 168 (1986) 838.

[16] Sze, S. M., Physics of Semiconductor Devices (J. Wiley) 1981

[17] Michaelson, S. B., J. Appl. Phys. 48 (1979) 4729.

[18] Barret, C., Vapaille, A., J. Appl. Phys. 50 (1979) 4217.

[19] Freeouf, J. L., proc. 2nd Int. Conf. on Surf. and Interf., Trieste (1982).

[20] Muret, P., Thèse d'Etat, Grenoble (1985).

[21] Muret, P., Deneuville, A., Surf. Sci. 168 (1986) 830.

[22] Newman, N., Van SchilfgaArde, M., Kendelewicz, T., Williams, M. D., Spicer, W. E., Phys. Rev. B 33 (1986) 1146.

[23] Barret, C., VAPAille, A., Solid-State Electron. 21 (1978) 1209.

[24] Chekir, F., Barret, C., Vapaille, A., J. Appl. Phys. 54 (1983) 6474.

[25] Chekir, F., Barret, C., Appl. Phys. Lett. 45 (1984) 1212.

[26] Spicer, W. E., Lindau, I., Skeath, P., Su, C. Y., Chye, P., Phys. Rev. Lett. 44 (1980) 420.

[27] Skeath, P., Su, S. Y., Hind, J., Lindau, I., SPICER, W. E., Appl. Phys. Lett. 39 (1981) 349. 